\title{
Propriedades Psicométricas da Versão Brasileira do The Coparenting Inventory for Parents and Adolescents (CI-PA)
}

\author{
Clarisse Pereira Mosmann¹, Mariana Rodrigues Machado, Crístofer Batista da Costa, \\ Pâmela Renata de Carvalho Gross, Daniel Abs \\ Universidade do Vale do Rio dos Sinos - Unisinos, São Leopoldo-RS, Brasil
}

\section{RESUMO}

Este estudo teve como objetivo examinar as propriedades psicométricas do The Coparenting Inventory for Parents and Adolescents (CI-PA) em uma amostra de 411 adolescentes com idades entre 11 a 18 anos $(M=14,44$ anos, $D P=1,86)$. Análises fatoriais confirmatórias foram realizadas por meio de modelagem de equações estruturais para testar a estrutura fatorial dessa amostra. A confiabilidade do inventário foi testada pelos coeficientes de alfa de Cronbach, variância media extraída e confiabilidade composta. E a invariância entre os grupos de meninos e meninas foi verificada por análises fatoriais confirmatórias multigrupos. Os resultados confirmaram uma estrutura de três fatores, com bom ajuste dos modelos com invariância entre os grupos analisados. A confiabilidade da escala apresentou valores adequados. Os testes realizados sugerem que a CI-PA apresenta propriedades psicométricas satisfatórias na população de adolescentes da amostra.

Palavras-chave: relações pais-filho, adolescente, adaptação, psicometria.

\begin{abstract}
Psychometric properties of the Brazilian version of The Coparenting Inventory for Parents and Adolescents (CI-PA)

This study aimed to examine the psychometric properties of a Brazilian version of The Coparenting Inventory for Parents and Adolescents (CI-PA) in a sample of 411 adolescents, from 11 to 18 years of age $(M=14.44$ years, $S D=1.86)$. Confirmatory Factor Analyses were performed through Structural Equations Modeling to test the factorial structure of the sample. The reliability of the inventory was tested by Cronbach's Alpha coefficients, Extracted Mean Variance and Composite Reliability. And the invariance between groups of boys and girls was verified by Multigroup Confirmatory Factor Analysis. Results confirmed a three-factor structure, with good fit of the models with invariance between the groups analyzed. The reliability of the scale presented adequate values. The tests performed suggest that CI-PA has satisfactory psychometric properties in the adolescent population of the sample.

Keywords: parent-child relationships; adolescent; adaptation; psychometry.
\end{abstract}

RESUMEN - Propiedades psicométricas de la versión brasileña del The Coparenting Inventory for Parents and Adolescents (CI-PA)

Este estudio tuvo como objetivo examinar las propiedades psicométricas del The Coparenting Inventory for Parents and Adolescents (CI-PA) en una muestra de 411 adolescentes con edades comprendidas entre 11 y 18 años $(M=14,44$ años, $D P=1,86)$. Análisis Factoriales Confirmatorios fueron realizados a través del Modelo de Ecuaciones Estructurales para testar la estructura factorial de esta muestra. La confiabilidad del inventario fue testada por los coeficientes Alpha de Cronbach, Varianza Extraída Media y Fiabilidad Compuesta. Y la invarianza entre los grupos de niños y niñas fue verificada por Análisis Factoriales Confirmatorios Multigrupos. Los resultados confirmaron una estructura de tres factores, con buen ajuste de los modelos con invarianza entre los grupos analizados. La confiabilidad de la escala presentó valores adecuados. Los test realizados señalan que la CI-PA presenta propiedades psicométricas satisfactorias en la población de adolescentes de la muestra.

Palabras clave: relaciones padres-hijo; adolescente; adaptación; psicometría.

A coparentalidade possui características e funções específicas que envolvem o processo relacional entre uma determinada díade (Baker, McHale, Strozier, \& Cecil, 2010). Trata-se de um subsistema constituído por uma dupla de cuidadores, não necessariamente os pais (Marsanic \& Kusmic, 2013), corresponsáveis pelos cuidados referentes à educação, proteção e desenvolvimento social e cognitivo de uma criança. Não se consideram questões emocionais, sexuais ou legais da conjugalidade, por exemplo, mas apenas o envolvimento da díade no que se refere à prole (Feinberg, 2003). 
Uma revisão sistemática da literatura sobre pesquisas enfocando o subsistema coparental apontou que estudos nacionais e internacionais têm reunido resultados relevantes para o trabalho na clínica psicológica e evidenciado a mediação da coparentalidade nas interrelações familiares (Costa, Machado, Schneider, \& Mosmann, 2017). Em outras palavras, por meio do subsistema coparental, problemas da relação conjugal tendem a reverberar na parentalidade, impactando a relação entre pais e filhos e no funcionamento de todo sistema familiar (Margolin, Gordis, \& John, 2001; Mosmann, Costa, Einsfeld, Silva, \& Koch, 2017; Mosmann, Costa, Silva, \& Luz, 2018).

Observar, avaliar e compreender empiricamente fenômenos e relações tão imbricados suscita a necessidade de aumentar cada vez mais o rigor metodológico nos procedimentos de pesquisa e no aprimoramento dos instrumentos utilizados (Frizzo, Kreutz, Schmidt, Piccinini, \& Bosa, 2005). O interesse pelo desenvolvimento de escalas que mensuram a coparentalidade surgiu inicialmente no final dos anos 90 com o Parenting Alliance Measure. Desde então, diversos instrumentos foram desenvolvidos com o objetivo de contribuir para a avaliação da relação coparental (Carvalho \& Barham, 2016).

Com o objetivo de identificar e comparar instrumentos de avaliação da coparentalidade, um estudo nacional de revisão sistemática analisou 35 estudos, entre os quais identificou oito escalas que têm sido utilizadas para medir a coparentalidade (Carvalho \& Barham, 2016). A qualidade das medidas foi avaliada por meio da análise das seguintes características: consistência interna, validade de conteúdo, estrutura interna, validade convergente, validade discriminante, validade de critério e evidências baseadas no processo de resposta. Três escalas apresentaram maior número de evidências relacionadas à qualidade: Parenting Alliance Measure (PAM), Coparenting Relationship Scale (CRS), e Coparenting Inventory for Parents and Adolescents (CI-PA). Segundo os autores, até 2016 não haviam sido encontradas medidas para avaliar o construto na população brasileira.

Portanto, mostra-se necessário dispor de instrumentos capazes de mensurar a coparentalidade e avaliar as possíveis mudanças decorrentes da participação de cuidadores em intervenções focadas na função coparental (Böing \& Crepaldi, 2016; Carvalho \& Barham, 2016; Souza, Ramos, Pontes, \& Silva, 2016; Teubert \& Pinquart, 2011a;). Ainda, quando se analisa instrumentos disponíveis para prática clínica, é necessário que o profissional tenha disponível ferramentas que o auxiliem a avaliar o desenvolvimento social, cognitivo e emocional de um indivíduo, bem como o contexto em que está inserido, como o funcionamento da dupla de cuidadores a partir das características da coparentalidade (McDaniel \& Teti, 2012).

Nesse sentido, o The Coparenting Inventory for Parents and Adolescents (CI-PA), diferencia-se por avaliar o exercício da função coparental sob diferentes perspectivas.
O inventário possui duas versões, uma para adolescentes e outra para cuidadores, e avalia a coparentalidade em dois níveis, individual e diádico. A versão respondida pelos adolescentes visa conhecer a percepção destes sobre a contribuição individual de cada um dos pais no que se refere ao exercício da coparentalidade e pela dupla em conjunto. $\mathrm{Na}$ versão respondida pelo casal coparental, inicia-se pela perspectiva individual, em que cada membro da dupla coparental se autoavalia, ponderando sobre a sua contribuição na coparentalidade e as características da sua participação. Em nível diádico, a dupla responde sobre o exercício conjunto dos cuidados e responsabilidades com o adolescente (Teubert \& Pinquart, 2011a).

O inventário contém três subescalas; cooperação, conflito e triangulação, cada uma com quatro itens pontuados em uma escala Likert de quatro pontos que variam de zero, se a afirmação é totalmente falsa, até quatro se a afirmação é totalmente verdadeira (Teubert \& Pinquart, 2011a). A cooperação é a dimensão que avalia o apoio/suporte, o respeito e a comunicação entre a dupla de cuidadores. Envolve a troca de informações sobre o adolescente e pressupõe um contexto relacional de confiança e lealdade entre a díade. A dimensão de conflito refere-se à divergência entre a dupla frente às demandas da adolescência. Envolve os princípios que orientam as decisões sobre a prole e os objetivos dos cuidadores. Por fim, a triangulação é a dimensão que mede o envolvimento do adolescente em situações de conflito coparental, configurando uma coalizão. Ocorre quando um membro da díade cria aliança com o adolescente para denegrir a imagem do outro membro ou sabotar a relação parental deste com o adolescente (Margolin et al., 2001; Teubert \& Pinquart, 2010a).

Nessa perspectiva, medidas de avaliação da coparentalidade podem ser excelentes ferramentas para pesquisadores e profissionais (Carvalho e Barham, 2016; Souza et al.,2016). O CI-PA, por ser um inventário que considera a avaliação dos cuidadores, mas também a percepção dos adolescentes, em dois níveis de análise, busca abarcar a complexidade das interações e, por isso, mostra-se particularmente relevante para mensurar o construto da coparentalidade.

A diferença na avaliação de adolescentes e cuidadores foi observada no estudo de Teubert e Pinquart (2010b), quando os autores utilizaram o CI-PA para avaliar as percepções dos pais e dos filhos adolescentes sobre a coparentalidade. Foram avaliadas 348 famílias (pais e filhos adolescentes) quanto a sintomatologia e a coparentalidade. Os resultados indicam que ao comparar a percepção da coparentalidade do filho com a dos pais percebe-se discrepâncias de caráter negativo, ou seja, os filhos avaliam negativamente a coparentalidade, apresentando um tamanho de efeito alto. Em contrapartida, quando ocorrem discrepâncias positivas entre pais e filhos, os tamanhos de efeito variam de pequenos à médios. 
Segundo alguns autores, adolescentes tendem a dar respostas mais fidedignas acerca dos estilos parentais de cada membro da díade (Kuppens, Grietens, Onghena, \& Michiels, 2009), da dinâmica familiar e, principalmente, dos conflitos conjugais (Teubert \& Pinquart, 2011a). A avaliação dos pais tende a ser diferente da avaliação dos adolescentes, já que estes últimos têm uma percepção mais crítica do contexto, enquanto os pais estão propensos a dar respostas com viés de desejabilidade social, já que avaliam a própria parentalidade e coparentalidade (Teubert \& Pinquart, 2010b; Teubert \& Pinquart, 2011a).

Outro estudo utilizando o The Coparenting Inventory for Parents and Adolescents (CI-PA) avaliou a ligação entre coparentalidade e variáveis da parentalidade e satisfação de vida do adolescente (Teubert \& Pinquart, 2011b). A amostra foi constituída de 476 famílias (pai, mãe e filho adolescente) de escolas públicas alemãs. Somente as subescalas de conflito e cooperação foram utilizadas no estudo, apresentando alfas de Cronbach de $\alpha=0,74$ e 0,87 , respectivamente. De acordo com os resultados, o conflito coparental na percepção dos cuidadores se associou aos baixos níveis de satisfação de vida na percepção do adolescente, enquanto a cooperação coparental se associou com altos níveis de satisfação de vida do adolescente.

Há significativa produção científica sobre coparentalidade na literatura internacional, porém, escassez de estudos no contexto brasileiro (Costa et al., 2017), indicando a necessidade de mais investigações (Böing \& Crepaldi, 2016). Além disso, a partir da revisão sistemática de Carvalho e Barham, (2016) até o ano de 2016 verificou-se que não existiam medidas de coparentalidade validadas para a população brasileira. Recentemente, está disponível a adaptação transcultural da Coparenting Relationship Scale (Carvalho, Barha, Souza, Böing, Crepaldi, \& Vieira, 2018), portanto, um primeiro estudo sobre o construto no Brasil. Esses dados sugerem que a escassez de ferramentas acessíveis e validadas ao contexto brasileiro podem interferir na realização e na qualidade das pesquisas desenvolvidas. Por isso, o presente estudo objetivou traduzir e examinar as propriedades psicométricas, consistência interna e validade de construto, do The Coparenting Inventory for Parents and Adolescents (CIPA) em uma amostra de adolescentes.

\section{Método}

\section{Participantes}

Participaram deste estudo 411 adolescentes, sendo 194 meninos (47,2\%), e 217 meninas (52,8\%). A coleta foi realizada por conveniência em escolas públicas (73\% dos adolescentes) e privadas (23\%) do interior do Rio Grande do Sul. Foram incluídos adolescentes que tinham entre 11 a 18 anos $(M=14,44$ anos, $D P=1,86)$ e que residiam com pelo menos um dos pais e/ou responsáveis. Foram excluídos adolescentes que não apresentaram o Termo de Consentimento Livre e Esclarecido
(TCLE) e o Termo de Assentimento Livre e Esclarecido (TALE) assinado.

\section{Instrumentos}

Questionário de dados sociodemográfico. Constituído de 24 questões, referente a dados sociodemográficos como: sexo, idade, escolaridade, cidade, número de irmãos, etc.

The Coparenting Inventory for Parents and Adolescents CI-PA (Teubert, \& Pinquart, 2011a). Neste estudo, foi utilizada a versão na qual o informante é o adolescente e avalia a díade coparental, as contribuições da mãe e contribuições do pai em cada escala com três subescalas (cooperação, conflito e triangulação), cada uma contando com quatro itens. Os itens são pontuados em uma escala Likert que varia de zero a quatro pontos, sendo: nada "verdadeiro" $=4$, "pouco verdadeiro" $=3$, "verdadeiro" $=2$, "muito verdadeiro" $=1$, "totalmente verdadeiro" $=0$.

O estudo original avaliou 528 adolescentes na Alemanha e apresentou alfas de Cronbach para díade, coparentalidade da mãe e coparentalidade do pai para cooperação de $\alpha=0,81,0,72,0,77$, para conflito $\alpha=0,74$; 0,$72 ; 0,77$, e para triangulação de $\alpha=0,75 ; 0,77 ; 0,79$ respectivamente. As análises fatoriais confirmatórias apresentaram os seguintes valores: (a) modelo diádico $\left(\chi^{2}=51, p<0,000 ; 1,84, \mathrm{RMSEA}=0,040 ; \mathrm{SRMR}=0,05 \mathrm{e}\right.$ $\mathrm{CFI}=0,97)$; (b) modelo reportando coparentalidade da mãe $\left(\chi^{2}=62, p<0,000\right.$; RMSEA $=0,058$; SRMR $=0,05$; CFI 0,94); e (c) modelo reportando a coparentalidade do pai $\left(\chi^{2}=62, p<0,000 ; 3,11\right.$, RMSEA $=0,063$; $\mathrm{SRMR}=0,05$ e CFI $=0,93$ ).

$\mathrm{O}$ processo de adaptação do instrumento para o português brasileiro seguiu as cinco etapas orientadas por Beaton, Bombardier, Guillemin e Ferraz, (2000), sendo elas: tradução, síntese, retrotradução, comitê de especialistas e pré-teste. $\mathrm{Na}$ etapa de tradução, os juízes entenderam que a apresentação original da pontuação das respostas na escala Likert poderia confundir o público adolescente, e levá-lo a erros de interpretação. Dessa forma, optou-se pela inversão da pontuação organizando-a da seguinte forma: "nada verdadeiro" $=0$, "pouco "verdade $r o "=1$, "verdadeiro igual" $=2$, "muito verdadeiro" $=3$, "totalmente verdadeiro" $=4$.

\section{Procedimentos Éticos e de Coleta de Dados}

O estudo foi aprovado sob número 14/152 (CAAE:36888214.0.0000.5344) pelo Comitê de Ética em Pesquisa da Universidade do Vale do Rio dos Sinos, conforme a Resolução 466/12 do Conselho Nacional de Saúde, que regulamenta a pesquisa com seres humanos no Brasil. Todos os adolescentes assinaram um Termo de Assentimento Livre e Esclarecido e seus pais assinaram um Termo de Consentimento Livre e Esclarecido. A aplicação foi realizada nas escolas de forma coletiva, em um encontro com duração de 90 minutos. Aos adolescentes foi assegurada a confidencialidade de todas as 
informações, que a participação ocorreria de maneira voluntária e a desistência dos participantes seria aceita em qualquer momento da pesquisa.

\section{Análise de Dados}

Inicialmente foram realizadas estatísticas descritivas quanto às médias e desvio padrão apresentadas pelos adolescentes nos fatores indicados na escala original (Teubert \& Pinquart, 2011a). Análises de Correlações de Pearson (Field, 2009) entre os itens das escalas e entre os fatores foram executadas. Para estabelecer as propriedades psicométricas da versão brasileira do CI-PA, foram realizadas análises fatoriais confirmatórias (AFC) a fim de verificar a estrutura fatorial na amostra pesquisada. As AFCs fazem parte do grupo de estatísticas que compõem as modelagens de equações estruturais (Structural Equation Modeling - SEM). Estas são análises que compreendem o estabelecimento de um modelo de relações entre variáveis observadas e variáveis latentes (fatores) a priori. Essas relações são pré-estabelecidas por estudos anteriores ou por modelos teóricos plausíveis para o fenômeno estudado. Para a realização das análises, são produzidas matrizes de variância-covariância das relações estipuladas e estas são comparadas à matriz de variância-covariância dos dados da amostra coletada (Blunch, 2008; Byrne, 2001; Coenders, Batista-Foguet, \& Saris, 2005; Hair, Black, Babin, Anderson, \& Tatham, 2009).

A aderência e ajuste do modelo previsto junto aos dados coletados indicam a qualidade da estrutura da escala e são indicadores psicométricos da validade de constructo da escala. Foram utilizados como índices desse ajuste o qui-quadrado $\left(\chi^{2}\right)$, o Comparative Fit Index (CFI), o Non-Normed Fit Index (NNFI) e o Root Mean Square Error of Approximation (RMSEA) com intervalo de confiança de $90 \%$. Como método de estimação, foi utilizado o Maximum Likelihood (ML). Os indicadores de um bom ajuste do modelo se referem a menores valores de qui-quadrado, a valores de CFI e NNFI superiores a 0,900 e preferencialmente superiores a 0,950 e a valores de RMSEA menores que 0,08 , sendo desaconselhável que o intervalo de confiança supere essa marca (Blunch, 2008; Coenders et al., 2005).

Análises fatoriais confirmatórias multigrupos ML também foram executadas para verificar a invariância de parâmetros entre os grupos de meninas e meninos para posterior uso da escala. Como o método de ML é especialmente sensível a normalidade da amostra, foi utilizada a estratégia de Bootstrap $(n=200)$ segundo as orientações de Coenders et al. (2005). Para análise da confiabilidade da escala, foram realizados o alfa de Cronbach, a variância média extraída (VME) e a confiabilidade composta da escala (CC), conforme as indicações de Hair et al. (2009).

\section{Resultados}

A Tabela 1 apresenta as médias, os desvio padrão e as correlações de Pearson das subescalas do CI-PA.

Tabela 1

Médias, Desvio Padrão e Correlações entre as Subescalas do CI-PA

\begin{tabular}{|c|c|c|c|c|c|c|c|c|c|c|c|}
\hline & Média & Desvio Padrão & 1 & 2 & 3 & 4 & 5 & 6 & 7 & 8 & 9 \\
\hline 1-CI-PA CP Díade & 11,45 & 3,93 & 1 & & & & & & & & \\
\hline 2-CI-PA CF Díade & 7,02 & 4,66 & $-0,79^{* *}$ & 1 & & & & & & & \\
\hline 3-CI-PA TR Díade & 2,10 & 3,31 & $-0,21^{* *}$ & $0,14^{* *}$ & 1 & & & & & & \\
\hline 4-CI-PA CP Mãe & 12,16 & 5,82 & $0,70^{* *}$ & $-0,68^{* *}$ & $-0,14^{* *}$ & 1 & & & & & \\
\hline 5-CI-PA CF Mãe & 6,00 & 5,31 & 0,04 & $-0,06$ & 0,03 & 0,09 & 1 & & & & \\
\hline 6-CI-PA TR Mãe & 2,65 & 3,67 & $-0,13^{* *}$ & $0,10^{*}$ & $0,29^{* *}$ & $-0,14^{* *}$ & $0,17^{* *}$ & 1 & & & \\
\hline 7-CI-PA CP Pai & 12,62 & 5,64 & $0,65^{* *}$ & $-0,65^{* *}$ & $-0,15^{* *}$ & $0,79^{* *}$ & $0,13^{* *}$ & $-0,21^{* *}$ & 1 & & \\
\hline 8-CI-PA CF Pai & 1,66 & 2,97 & $-0,08$ & 0,09 & $0,36^{* *}$ & $-0,11^{*}$ & 0,03 & $0,51^{* *}$ & $-0,15^{* *}$ & 1 & \\
\hline 9-CI-PA TR Pai & 5,33 & 4,51 & $0,11^{*}$ & $-0,10^{*}$ & $0,27^{* *}$ & 0,07 & $-0,26^{* *}$ & 0,10 & 0,03 & $0,27^{* *}$ & 1 \\
\hline
\end{tabular}

Nota. $\mathrm{CP}=$ cooperação coparental; $\mathrm{CF}=$ conflito coparental; $\mathrm{TR}=$ triangulação coparental

\section{Análises Fatoriais Confirmatórias}

Inicialmente foram estabelecidos e testados modelos para as subescalas conforme as indicações originais da literatura (Teubert \& Pinquart, 2011a). Os modelos relativos à coparentalidade da mãe e à coparentalidade do pai tiveram que ser ajustados quanto ao acréscimo de covariância entre erros e a supressão da covariância entre os fatores "cooperação" e "conflito" que não se mostrou estatisticamente significativa em ambos os modelos. Os modelos finais para essas duas subescalas foram novamente testados e os resultados estão expressos na Tabela 2.

Os pesos padronizados dos itens em relação aos fatores das subescalas, com intervalo de confiança de $90 \%$, são apresentados na Tabela 3 . 
Tabela 2

Análise Fatorial Confirmatória das Subescalas do CI-PA

\begin{tabular}{lcccccc}
\hline \multicolumn{1}{c}{ Modelo } & $\chi^{2}$ & $d f$ & $p$ & NNFI & CFI & RMSEA (90\%) \\
\hline Mãe Inicial & 196,21 & 62 & $<0,001$ & 0,913 & 0,931 & $0,073(0,031-0,084)$ \\
Mãe Final & 159,41 & 62 & $<0,001$ & 0,937 & 0,950 & $0,062(0,050-0,074)$ \\
Pai Inicial & 330,53 & 62 & $<0,001$ & 0,863 & 0,891 & $0,103(0,092-0,114)$ \\
Pai Final & 135,84 & 60 & $<0,001$ & 0,960 & 0,969 & $0,055(0,043-0,068)$ \\
Díade & 157,78 & 51 & $<0,001$ & 0,953 & 0,963 & $0,069(0,057-0,082)$ \\
\hline
\end{tabular}

Tabela 3

Pesos Padronizados Subescalas (Bootstrap; ML; $n=200$; IC=90\%)

\begin{tabular}{|c|c|c|c|c|c|}
\hline \multicolumn{3}{|c|}{ Item <--- Fator } & \multirow{2}{*}{$\begin{array}{c}\text { Estimado } \\
0,611\end{array}$} & \multirow{2}{*}{$\begin{array}{c}\text { Mín } \\
0,539\end{array}$} & \multirow{2}{*}{$\begin{array}{c}\text { Máx } \\
0,677\end{array}$} \\
\hline Mãe Item 4 & $<---$ & cooperação & & & \\
\hline Mãe Item 3 & $<---$ & cooperação & 0,667 & 0,608 & 0,727 \\
\hline Mãe Item 2 & $<---$ & cooperação & 0,901 & 0,872 & 0,928 \\
\hline Mãe Item 1 & $<---$ & cooperação & 0,881 & 0,847 & 0,914 \\
\hline Mãe Item 5 & $<---$ & cooperação & 0,549 & 0,467 & 0,612 \\
\hline Mãe Item 9 & $<---$ & conflito & 0,620 & 0,521 & 0,836 \\
\hline Mãe Item 8 & $<---$ & conflito & 0,283 & 0,186 & 0,763 \\
\hline Mãe Item 7 & $<---$ & conflito & 0,727 & 0,537 & 0,828 \\
\hline Mãe Item 6 & $<---$ & conflito & 0,581 & 0,388 & 0,643 \\
\hline Mãe Item 13 & $<---$ & triangulação & 0,562 & 0,423 & 0,681 \\
\hline Mãe Item 12 & $<---$ & triangulação & 0,752 & 0,673 & 0,832 \\
\hline Mãe Item 11 & $<---$ & triangulação & 0,930 & 0,885 & 0,977 \\
\hline Mãe Item 10 & $<---$ & triangulação & 0,677 & 0,622 & 0,735 \\
\hline Pai Item 4 & $<---$ & cooperação & 0,772 & 0,710 & 0,819 \\
\hline Pai Item 3 & $<---$ & cooperação & 0,818 & 0,762 & 0,863 \\
\hline Pai Item 2 & $<---$ & cooperação & 0,656 & 0,581 & 0,713 \\
\hline Pai Item 1 & $<---$ & cooperação & 0,681 & 0,610 & 0,748 \\
\hline Pai Item 5 & $<---$ & cooperação & 0,624 & 0,544 & 0,689 \\
\hline Pai Item 9 & $<---$ & conflito & 0,843 & 0,777 & 0,895 \\
\hline Pai Item 8 & $<---$ & conflito & 0,808 & 0,748 & 0,860 \\
\hline Pai Item 7 & $<---$ & conflito & 0,556 & 0,476 & 0,633 \\
\hline Pai Item 6 & $<---$ & conflito & 0,496 & 0,422 & 0,566 \\
\hline Pai Item 13 & $<---$ & triangulação & 0,760 & 0,654 & 0,853 \\
\hline Pai Item 12 & $<---$ & triangulação & 0,970 & 0,924 & 1,000 \\
\hline Pai Item 11 & $<---$ & triangulação & 0,790 & 0,683 & 0,880 \\
\hline Pai Item 10 & $<---$ & triangulação & 0,529 & 0,413 & 0,646 \\
\hline Díade Item 4 & $<---$ & cooperação & 0,871 & 0,835 & 0,899 \\
\hline Díade Item 3 & $<---$ & cooperação & 0,137 & 0,042 & 0,237 \\
\hline Díade Item 2 & $<---$ & cooperação & 0,883 & 0,850 & 0,909 \\
\hline Díade Item 1 & $<---$ & cooperação & 0,849 & 0,806 & 0,889 \\
\hline Díade Item 8 & $<---$ & conflito & 0,766 & 0,719 & 0,806 \\
\hline Díade Item 7 & $<---$ & conflito & 0,916 & 0,893 & 0,941 \\
\hline Díade Item 6 & $<---$ & conflito & 0,768 & 0,727 & 0,809 \\
\hline Díade Item 5 & $<---$ & conflito & 0,543 & 0,457 & 0,611 \\
\hline Díade Item 12 & $<---$ & triangulação & 0,617 & 0,502 & 0,698 \\
\hline Díade Item 11 & $<---$ & triangulação & 0,710 & 0,633 & 0,778 \\
\hline Díade Item 10 & $<---$ & triangulação & 0,916 & 0,860 & 0,960 \\
\hline Díade Item 9 & $<---$ & triangulação & 0,752 & 0,666 & 0,831 \\
\hline
\end{tabular}


As análises multigrupo foram realizadas para verificar a invariância métrica, escalar e de configuração entre meninos e meninas, conforme as orientações de Byrne (2001) e Blunch (2008). Os autores indicam que entre um modelo e outro, para que se assuma a invariância estrutural entre os grupos, é aceitável uma diferença de no máximo 0,010 no índice de ajuste CFI e ausência de diferença significativa entre os $\chi^{2}$ dos modelos. Foi verificado que é possível assumir a invariância de parâmetros entre meninos e meninas na amostra, conforme indica a Tabela 4, o que é um dado relevante para futuros usos da escala.

Tabela 4

Análise Fatorial Confirmatória Multigrupo

\begin{tabular}{lcccccc}
\hline \multicolumn{1}{c}{ Modelo } & $\chi^{2}$ & $d f$ & $p$ & NNFI & CFI & RMSEA (90\%) \\
\hline Mãe Configural & 250,91 & 124 & $<0,001$ & 0,922 & 0,938 & $0,050(0,041-0,059)$ \\
Mãe Métrica & 268,53 & 134 & $<0,001$ & 0,923 & 0,934 & $0,050(0,041-0,058)$ \\
Mãe Escalar & 291,33 & 147 & $<0,001$ & 0,925 & 0,929 & $0,049(0,041-0,057)$ \\
Pai Configural & 196,96 & 120 & $<0,001$ & 0,960 & 0,969 & $0,040(0,029-0,049)$ \\
Pai Métrica & 213,10 & 130 & $<0,001$ & 0,960 & 0,967 & $0,040(0,030-0,049)$ \\
Pai Escalar & 234,27 & 143 & $<0,001$ & 0,961 & 0,964 & $0,040(0,030-0,048)$ \\
Díade Configural & 240,37 & 102 & $<0,001$ & 0,936 & 0,951 & $0,058(0,048-0,067)$ \\
Díade Métrica & 248,73 & 111 & $<0,001$ & 0,941 & 0,951 & $0,055(0,046-0,064)$ \\
Díade Escalar & 258,24 & 123 & $<0,001$ & 0,948 & 0,952 & $0,052(0,043-0,061)$ \\
\hline
\end{tabular}

\section{Confiabilidade e Variância Média Extraída}

Considerando a confiabilidade da escala no geral, os resultados foram bastante satisfatórios. Foram analisados o alfa de Cronbach, a medida da confiabilidade composta, e a variância média extraída dos fatores das subescalas do CI-PA. Os valores de alfa encontrados são semelhantes ao da escala original (Teubert \& Pinquart, 2011a), conforme Tabela 5, com exceção do valor para conflito da mãe, que apresentou valor considerado abaixo do esperado tanto para o alfa $(\alpha=0,63)$ quanto na confiabilidade composta, apesar de que o valor original $(\alpha=0,69)$ também era limítrofe ao indicado de 0,70. A VME para esse fator também ficou abaixo do recomendado que é de 0,500 . Os dados que indicam pouca convergência nesse fator talvez sejam devidos provavelmente à influência do item 8 (Minha mãe acha que meu pai dá muita liberdade pra mim) que se apresentou deslocado na escala, com peso padronizado estimado baixo, e com valores entre 0,186 e 0,763 , o que seria uma grande variabilidade para o intervalo de confiança estipulado em $90 \%$, além de correlações de Pearson de baixa intensidade $(r<0,20)$ e somente com os itens 6 e 7.

Tabela 5

Confiabilidade Composta, Alfa de Cronbach e Variância Média Extraída dos Fatores das Subescalas do CI-PA

\begin{tabular}{|c|c|c|c|c|c|c|c|c|c|}
\hline & \multicolumn{3}{|c|}{ Mãe } & \multicolumn{3}{|c|}{ Pai } & \multicolumn{3}{|c|}{ Díade } \\
\hline & $\alpha$ & CC & VME & $\alpha$ & CC & VME & $\alpha$ & $\mathrm{CC}$ & VME \\
\hline Conflito & 0,63 & 0,647 & 0,333 & 0,79 & 0,778 & 0,480 & 0,84 & 0,841 & 0,578 \\
\hline Triangulação & 0,81 & 0,826 & 0,551 & 0,86 & 0,855 & 0,605 & 0,83 & 0,840 & 0,572 \\
\hline Cooperação & 0,86 & 0,850 & 0,542 & 0,85 & 0,837 & 0,510 & 0,75 & 0,814 & 0,569 \\
\hline
\end{tabular}

\section{Discussão}

O presente estudo teve como objetivo traduzir e apresentar propriedades psicométricas do CI-PA, em uma amostra brasileira de adolescentes com idades entre 11 e 18 anos. Para tal, inicialmente, realizou-se o processo de tradução e retrotradução e, posteriormente, análises descritivas e inferenciais, como correlações de Pearson, análises fatoriais confirmatórias multigrupo e análises de confiabilidade da escala, de forma a testar a estrutura com três fatores latentes (cooperação, triangulação e conflito coparental), avaliando a coparentalidade da mãe, do pai e da díade, pela perspectiva do adolescente.

Os resultados foram bastante satisfatórios no que diz respeito às relações entre os itens e quanto à estrutura fatorial da escala, pois os modelos apresentaram índices de ajuste considerados bons pela literatura, indicando que a estrutura fatorial proposta teve aderência à amostra pesquisada. Os dados referentes as análises multigrupo indicaram que a escala possui equivalência configural, 
métrica e escalar, o que indica qualidade para ser utilizada tanto na população masculina quanto feminina, não apresentando diferenças expressivas quanto ao entendimento desses dois grupos adolescentes em relação ao significado dos construtos abordados e aos pesos que cada item tem com relação a cada construto. Essa informação é relevante pois permite comparar diferentes grupos de meninos e meninas quanto às médias de cada grupo de melhor forma, pois diferenças entre essas médias não seriam atribuíveis as diferenças na compreensão e na estrutura fatorial da escala.

Os valores de alfa, confiabilidade composta e de variância média extraída foram convergentes na indicação da boa qualidade da escala, de uma forma geral. Ao comparar os dados obtidos com o estudo original os valores de alfa da amostra brasileira apresentaram melhores valores excetuando os fatores de conflito coparental da mãe e cooperação coparental da díade (Teubert \& Pinquart, 2011a). Destaca-se o valor do fator conflito na avaliação da coparentalidade da mãe, indicando que provavelmente deveria ser repensado o uso do item 8 na composição da escala para esse público.

Para o público adolescente brasileiro, a escala apresentou boas condições para sua aplicação e uso, corroborando a avaliação feita por Carvalho e Barham (2016) acerca da qualidade do The Coparenting Inventory for Parents and Adolescents. Os principais cuidados devem se referir a inferências, utilizando o fator conflito na avaliação da coparentalidade da mãe, tendo o item 8 presente. Destacase que esta pesquisa vem suprir o déficit de instrumentos em português que avaliam o subsistema coparental na perspectiva do adolescente, disponibilizando para pesquisadores e clínicos brasileiros uma escala que mensura três fatores da coparentalidade, sendo eles os níveis de cooperação entre os pais, conflito e triangulação.

Pode ser apontado como limitações deste estudo a não utilização de outras estratégias de busca de evidências de validade para a escala. Por exemplo, avaliação da validade de conteúdo e de critério por meio da comparação dos resultados obtidos com os de outra escala com medida equivalente do construto. Além disso, verificar a consistência da medida por meio de aplicações com intervalo de tempo de dias ou semanas possibilitaria avaliar a estabilidade temporal da medida.

Além disso, indica-se a replicação da presente pesquisa com outros grupos com características e em contextos distintos e com um número maior de participantes, procedimentos que garantem maior fidedignidade $\mathrm{e}$ validade de mensuração da escala. Finalmente, sugere-se que estudos sejam conduzidos a fim de validar a escala de avaliação da coparentalidade na perspectiva dos pais e cuidadores. Embora o objetivo deste estudo tenha sido avaliar somente a percepção da coparentalidade pelos adolescentes, medidas de coparentalidade na percepção dos pais tornará a avaliação do fenômeno mais efetiva considerando, principalmente, a complexidade das interações entre o subsistema conjugal, parental e coparental, tendo em vista que há diferenças de percepção sobre o fenômeno da coparentalidade entre eles (Teubert \& Pinquart, 2010b; Teubert \& Pinquart, 2011a).

\section{Referências}

Baker, J., McHale, J., Strozier, A., \& Cecil, D. (2010). Mother-grandmother coparenting relationships in families with incarcerated mothers: A pilot investigation. Family Process, 49(2), 165-184. doi:10.1111/j.1545-5300.2010.01316.x

Beaton, D. E., Bombardier, C., Guillemin, F., \& Ferraz, M. B. (2000). Guidelines for the process of cross-cultural adaptation of self-report measures. Spine, 25(24), 3186-3191. doi: 10.1097/00007632-200012150-00014

Blunch, N. (2008). Introduction to structural equation modeling using IBM SPSS statistics and AMOS. CA: Sage.

Böing, E., \& Crepaldi, M. A. (2016). Relação pais e filhos: Compreendendo o interjogo das relações parentais e coparentais. Educar Em Revista, 59(1), 17-33. doi: 10.1590/0104-4060.44615

Byrne, B. M. (2001). Structural equation modeling with AMOS: Basic concepts, applications, and programming. New Jersey: LEA.

Carvalho, T. R., Barha, E. J., Souza, C. D., Böing, E., Crepaldi, M. A., \& Vieira, M. L. (2018). Cross-cultural adaptation of an instrument to assess coparenting: Coparenting Relationship Scale. Psico-USF, 23(2), 215-227. doi: 10.1590/1413-82712018230203

Carvalho, T. R. D., \& Barham, E. J. (2016). Instrumentos para avaliar a coparentalidade: Uma comparação de suas propriedades psicométricas. Avaliação Psicológica, 15(2), 207-215. doi: 10.15689/ap.2016.1502.09

Coenders, G., Batista-Foguet, J. M., \& Saris, W. E. (2005). Temas avanzados em modelos de ecuaciones estructurales. Madrid: Muralla.

Costa, C. B., Machado, M. R., Schneider, M. C., \& Mosmann, C. P., (2017). Subsistema coparental: Revisão sistemática de estudos empíricos. Revista Psico, 48(4), 339-351. doi: 10.15448/1980-8623.2017.4.25386

Feinberg, M. E. (2003). The internal structure and ecological context of coparenting: A framework for research and intervention. Parenting: Science and Practice, 3(2), 95-131. doi: 10.1207/S15327922PAR0302

Field, A. (2009). Descobrindo a estatística usando o SPSS. Porto Alegre: Bookman.

Frizzo, G. B., Kreutz, C. M., Schmidt, C., Piccinini, C. A., \& Bosa, C. (2005). O conceito de coparentalidade e suas implicações para a pesquisa e para a clínica. Revista Brasileira de Crescimento e Desenvolvimento Humano, 15(3), 84-93. Recuperado de http://pepsic.bvsalud. org/pdf/rbcdh/v15n3/10.pdf

Hair, J. F., Black, W. C., Babin, B., J., Anderson, R. E., \& Tatham, R. L. (2009). Análise multivariada de dados. Porto Alegre: Bookman.

Kuppens, S., Grietens, H., Onghena, P., \& Michiels, D. (2009). Measuring parenting dimensions in middle childhood. European Journal of Psychological Assessment, 25(3), 133-140. doi: 10.1027/1015-5759.25.3.133 
Margolin, G., Gordis, E. B., \& John, R. S. (2001). Coparenting: A link between marital conflict and parenting in two parent families. Journal of family Psychology, 15(1), 3-21. doi: 10.1037/0893-3200.15.1.3

Marsanic, B. V., \& Kusmic, E. (2013). Coparenting within the family system: Review of literature. Coll. Antropol., 37(4), 1379-1383. Recuperado de https://goo.gl/WRGCyH

McDaniel, B. T., \& Teti, D. M. (2012). Coparenting during the first three months after birth: The role of infant sleep quality. Journal of Family Psychology, 26(6), 886-895. doi: 10.1037/a0030707

Mosmann, C. P., Costa, C. B., Einsfeld, P., Silva, A. G. M., \& Koch, C. (2017). Conjugalidade, parentalidade e coparentalidade: Associações com sintomas externalizantes e internalizantes em crianças e adolescentes. Estudos de Psicologia, 34(4), 487-498. doi: 10.1590/198202752017000400005

Mosmann, C. P., Costa, C. B., Silva, A. G. M., \& Luz., S. K. (2018). Filhos com sintomas psicológicos clínicos: Papel discriminante da conjugalidade, coparentalidade e parentalidade. Temas em Psicologia, 26(1), 429-442. doi: 10.9788/TP2018.1-17Pt

Souza, P. B. M. de, Ramos, M. D. S., Pontes, F. A. R., \& Silva, S. S. da C. (2016). Coparentalidade: Um estudo de revisão sistemática de literatura. Estilos da Clinica, 21(3), 700-720. doi: 10.11606/issn.1981-1624.v21i3p700-720

Teubert, D., \& Pinquart, M. (2010a). The association between coparenting and child adjustment: A meta-analysis. Parenting: Science and Practice, 10(4), 286-307. doi:10.80/15295192.2010.492040

Teubert, D., \& Pinquart, M. (2010b). Discrepancies in adolescents' versus parents' reports of coparenting: A cluster analytic approach. Family Science, 1(3-4), 183-190. doi:10.1080/19424620.2010.537960

Teubert, D., \& Pinquart, M. (2011a). The coparenting inventory for parents and adolescents (CI-PA). European Journal of Psychological Assessment, 27(3), 206-215. doi: 10.1027/1015-5759/a000068

Teubert, D., \& Pinquart, M. (2011b). The link between coparenting, parenting, and adolescent life satisfaction. Family Science, 2(4), $221-229$. doi: 10.1080/19424620.2012.666655

Teubert, D., \& Pinquart, M. (2010a). The association between coparenting and child adjustment: A meta-analysis. Parenting: Science and Practice, 10(4), 286-307. doi:10.80/15295192.2010.492040

Teubert, D., \& Pinquart, M. (2010b). Discrepancies in adolescents' versus parents' reports of coparenting: A cluster analytic approach. Family Science, 1(3-4), 183-190. doi:10.1080/19424620.2010.537960

\section{Sobre os autores}

Clarisse Pereira Mosmann é doutora em Psicologia (PUCRS) e professora do Programa de Pós-Graduação em Psicologia da Universidade do Vale do Rio dos Sinos.

Mariana Rodrigues Machado é doutoranda em Psicologia no Programa de Pós-Graduação em Psicologia da Universidade do Vale do Rio dos Sinos.

Crístofer Batista Costa é doutorando em Psicologia no Programa de Pós-Graduação em Psicologia da Universidade do Vale do Rio dos Sinos.

Pâmela Renata de Carvalho Gross é graduanda em Psicologia pela Universidade do Vale do Rio dos Sinos e bolsista UNIBIC de Iniciação Científica.

Daniel Viana Abs da Cruz é doutor em Psicologia (UFRGS) e professor da Graduação da Universidade do Vale do Rio dos Sinos. 